Universidad de Lima

Facultad de Psicología

Carrera de Psicología

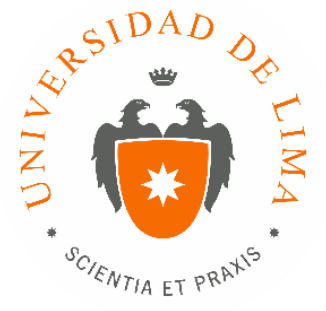

\title{
EXPERIENCIA DE TRABAJO EN EL DEPARTAMENTO DE PSICOLOGÍA DE UN CENTRO DE ATENCIÓN COMUNITARIA
}

Trabajo de suficiencia profesional para optar el título profesional de Licenciado en Psicología

\author{
Micaela de Las Casas Solf
}

Código 20081346

\author{
Lima - Perú
}

Febrero de 2019 



\section{EXPERIENCIA DE TRABAJO EN EL DEPARTAMENTO DE PSICOLOGÍA DE UN CENTRO DE ATENCIÓN COMUNITARIA}




\section{TABLA DE CONTENIDO}

INTRODUCCIÓN

CAPÍTULO I: IDENTIFICACIÓN DEL PROBLEMA ........................................................................8

CAPÍTULO II: DESCRIPCIÓN DE LAS ACTIVIDADES Y TAREAS REALIZADAS..............11

2.1 Intervenciones grupales en salud mental ................................................................11

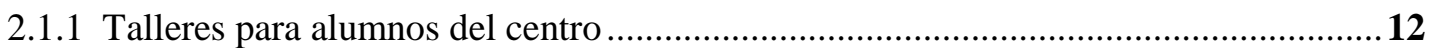

2.1.2 Taller para el manejo de emociones ...............................................................13

2.2 Intervenciones individuales.......................................................................................................... 14

CAPÍTULO III: RESULTADOS DE LA INTERVENCIÓN...............................................................16

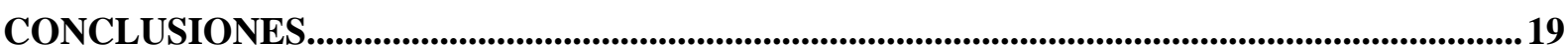

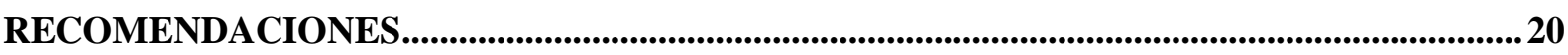

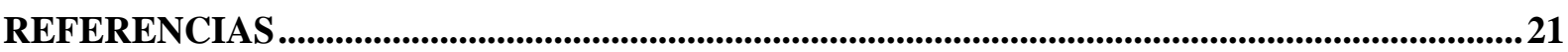

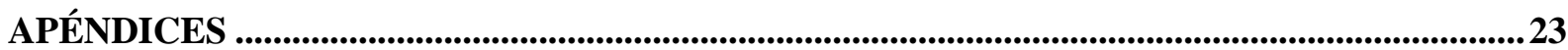




\section{ÍNDICE DE TABLAS}

Tabla A.: Esquema de la sesión con adolescentes sobre las relaciones sexuales y enfermedades de transmisión sexual.

Tabla C.1.: Ejemplo de análisis funcional de un caso 


\section{ÍNDICE DE APÉNDICES}

Apéndice A: Esquema de la sesión con adolescentes sobre las relaciones sexuales y enfermedades de transmisión sexual.

Apéndice B: Esquema del taller manejo de emociones.

Apéndice C: Ejemplo de análisis funcional de un caso... 


\section{INTRODUCCIÓN}

El presente trabajo describe las actividades realizadas en el Departamento de Psicología de un centro de atención comunitaria como voluntaria durante el periodo Agosto 2016 - Agosto 2018. Los talleres de prevención y las atenciones psicológicas individuales tuvieron como fin satisfacer las necesidades de asistencia en salud mental encontradas en la población usuaria del centro y el uso de los conocimientos adquiridos en la práctica de la psicología. Los trastornos y dificultades que tuvieron mayor ocurrencia fueron: a) trastornos depresivos; b) trastornos de ansiedad; c) problemas familiares; d) problemas conductuales en niños; e) trastornos de la personalidad; y f) situaciones de cambio. Cada caso siguió un proceso de evaluación y orientación de acuerdo a las necesidades y particularidades presentadas. El enfoque cognitivo conductual fue el que guió la mayoría de las intervenciones, las cuales fueron supervisadas y validadas por los psicólogos profesionales encargados del proyecto social. Los talleres se implementaron de acuerdo a las necesidades encontradas en los grupos objetivo. Se encontró que los estudiantes adolescentes del centro asistieron y participaron de los talleres en su totalidad. Sin embargo, los participantes en el taller del control de emociones presentaron irregularidades en su asistencia. Esta conducta fue más evidente en las intervenciones psicológicas individuales, en las cuales el $26.3 \%$ de las personas atendidas completaron los programas de intervención ofrecidos. Entre los motivos de las inasistencias de los usuarios atendidos se encontró que las dificultades o cambios inesperados en el trabajo, escuela y familia son algunos de los factores que más influyen en su participación y permanencia. 


\section{CAPÍTULO I: IDENTIFICACIÓN DEL PROBLEMA}

En el Perú, el sistema de salud presenta carencias significativas que dificultan el acceso a una atención adecuada, especialmente en las poblaciones vulnerables y de menor nivel educativo. De acuerdo con los datos del Censo Nacional del 2017 encontrados por el Instituto Nacional de Estadística e Informática (2018) 2 millones 357 mil 962 habitantes de la Provincia de Lima no cuentan con un seguro de salud. Asimismo, de estos, 1 millón 75 mil 310 peruanos tienen como máximo nivel educativo alcanzado la educación secundaria.

En cuanto a la atención en salud mental en el Perú:

Se estima que [hay] 4 millones de peruanos con alguna enfermedad mental, de los cuales solo el $20 \%$ recibe tratamiento adecuado. Esto se debe a que los ciudadanos no reconocen los problemas de salud mental como tales y a la falta de servicios adecuados para tratar estas enfermedades. (Instituto Integración de Análisis y Comunicación, 2016)

Es decir, que las fallas que presentan los servicios de salud, el desconocimiento y el estigma asociados a las enfermedades mentales son las causas más significativas del bajo número de personas que buscan y reciben atención adecuada. Una de estas deficiencias es la escasa oferta de servicios de salud mental cercanas al entorno cotidiano de la población. La Organización Panamericana de la Salud (2009), considera que "La estigmatización y discriminación de las personas con trastornos mentales es uno de los obstáculos más grandes que hay" (p.10). Debido a ello urge la necesidad de concientizar a la población mediante la promoción de la salud y la prevención de los trastornos psíquicos desde edades tempranas del desarrollo y en los diferentes entornos particulares en los que las personas se relacionan, siendo de gran relevancia la escuela.

Se conoce que las enfermedades mentales y los problemas que requieren de atención psicológica tienen diferentes índices de prevalencia en la población. En Perú, "los problemas 
de mayor prevalencia [son] la depresión, el abuso y dependencia del alcohol, los trastornos de ansiedad, las esquizofrenias, la violencia familiar, los trastornos de conducta y de las emociones en los niños y el maltrato infantil.” (Ministerio de Salud, 2018, p.13).

El distrito en el que se encuentra ubicado el centro y del cual proviene la principal población asistida en el centro comunitario, cuenta con una población de más de 355 mil personas (INEI, 2018) y es uno de los distritos más poblados tanto de la provincia de Lima, como del Perú. Asimismo, la mayoría de los vecinos viven organizados en agrupaciones vecinales de menor desarrollo y la incidencia de pobreza al 2013 por el INEI (2015) estuvo entre el rango de $19 \%-28 \%$ a nivel distrital.

Teniendo en consideración los datos presentados, las carencias del sistema de salud del país y las dificultades que evidencian las poblaciones en desventaja económica para obtener acceso a los servicios y al mantenimiento de la salud; el distrito presenta una demanda insatisfecha de instituciones y proyectos que brinden atención accesible en salud para sus habitantes.

Entre los objetivos del Departamento de Psicología están: a) la aplicación práctica de los conocimientos teóricos aprendidos; b) desarrollar en los psicólogos profesionales y voluntarios, por medio de su vocación de servicio, la responsabilidad social y la solidaridad; y c) brindar un servicio gratuito de atención psicológica mediante programas de intervención/prevención grupal e individual, del cual se beneficiarán los alumnos, los padres de familia, los profesores y la comunidad en general. En este se brindan los servicios de evaluación, orientación y consejería impartidos por psicólogos profesionales y voluntarios en materia de prevención e intervención en el ámbito de la salud mental.

En el Departamento de Psicología, durante el periodo de agosto del 2016 a diciembre del 2017, el servicio de atención fue diario. Sin embargo, a partir del 2018, el programa fue modificado tras ser reconocidas las necesidades de la población usuaria, la misión del Departamento de Psicología y los recursos disponibles del mismo. De esta forma, la 
intervención psicológica individual disminuyó y se implementaron los talleres de intervención grupales. Entre las dificultades identificadas con mayor frecuencia entre los usuarios fueron los trastornos depresivos, trastornos de ansiedad, problemas familiares, problemas conductuales en niños, trastornos de la personalidad y situaciones de cambio. Problemáticas que en su mayoría concuerdan con las encontradas como de mayor prevalencia en la población nacional por el Ministerio de Salud. 


\section{CAPÍTULO II: DESCRIPCIÓN DE LAS ACTIVIDADES Y TAREAS REALIZADAS}

Se presenta la descripción y el desarrollo de las principales tareas ejecutadas en el puesto de voluntaria en el Departamento de Psicología del centro comunitario. Siendo estas: Asistencia y desarrollo de intervenciones grupales en prevención y promoción en salud mental; y asistencia psicológica individual. Debido a que el fin de las acciones desempeñadas recae en la prevención, intervención y consulta en salud mental, tanto en las atenciones individuales como grupales, el trabajo responde al enfoque psicológico clínico-comunitario (Sánchez, 2007) bajo el cual se desarrolla este proyecto social. Asimismo, ambos tipos actividades se alinean con los objetivos, previamente presentados, del Departamento de Psicología.

\subsection{Intervenciones grupales en salud mental}

Los talleres grupales tienen el objetivo de promover la salud y prevenir los trastornos mentales en la comunidad. Giraldo, Toro, Macias, Valencia y Palacio (2010) reconocen que "Los conocimientos: determinan en gran medida la adopción de prácticas saludables, puesto que permiten a las personas tomar decisiones informadas y optar por prácticas saludables o de riesgo frente al cuidado de su salud" (p.137)

Se implementaron 3 talleres de diferente temática. Dos estuvieron orientados a los estudiantes de la institución y uno, a los jóvenes y adultos que solicitaron atención en el Departamento de Psicología que evidenciaron dificultades en el control de sus emociones. 


\subsubsection{Talleres para alumnos del centro}

El departamento de Psicología, tuvo como parte de sus actividades apoyar en el desarrollo integral de los estudiantes mediante la ejecución de talleres de prevención y promoción de la salud. Para los dos talleres, los temas a trabajar fueron escogidos en base a las a los intereses de los adolescentes de los tres últimos años de estudio y los planteados por el equipo del Departamento de Psicología. Entre estos estaban el consumo de alcohol y drogas, las relaciones sexuales, hábitos saludables y el rendimiento académico. Ambos fueron desarrollados bajo un enfoque educativo, participativo, colaborativo, dinámico y de reflexión.

El principal objetivo del taller sobre estilo de vida saludable fue el reconocer las actividades que lo promueven y cómo minimizar los riesgos. Se identificaron ocho alumnos adolescentes en situación de riesgo, para los cuales se diseñó un taller de estilo de vida saludable. Las sesiones del taller fueron implementadas por diferentes facilitadores del Departamento de Psicología.

En este caso, la sesión desarrollada fue acerca de las relaciones sexuales y las enfermedades de transmisión sexual. Por mucho tiempo la educación sexual fue impartida con un enfoque prohibicionista con un marcado valor en la abstinencia y la visión religiosa de llegar virgen al matrimonio. Sin embargo, “Una educación sexual completa comienza con la abstinencia, pero además toma en cuenta que muchos jóvenes van a decidir tener sexo, por lo tanto, tienen que conocer las consecuencias y la manera de protegerse a sí mismos.” (Ardila, 2007, p.83). El objetivo de la sesión fue el de sensibilizar a los adolescentes sobre las enfermedades de transmisión sexual, las vías de transmisión y conductas preventivas. La sesión se dividió en 4 actividades que fomentaron el 
aprendizaje, la participación y la reflexión de los adolescentes en estos temas. (Ver Apéndice A)

El segundo taller para estudiantes, se realizó en una sola sesión de dos horas y media. Se trabajó con 40 alumnos, que cursaban los dos últimos años de estudio, divididos en cinco grupos. El objetivo fue el reconocimiento de los factores facilitadores del consumo de drogas, los riesgos del abuso de las mismas y las conductas responsables durante el consumo. Con el grupo de trabajo al que se lideró se buscó que los temas y las actividades partieran de sus propios conocimientos y vivencias, en virtud de generar una mayor motivación y un aprendizaje más significativo. Para lograrlo, se fomentó el intercambio de experiencias y conocimientos entre los adolescentes, considerando la importancia de los pares durante la adolescencia y su influencia en las actitudes hacia las drogas (Calero, Schmidt y Bugallo, 2016; Lavado, Aragón, y Gonzales, 2015)

\subsubsection{Taller para el manejo de emociones}

El objetivo del taller fue el de aprender técnicas para el control de las emociones. Se desarrolló, utilizando la teoría y las herramientas de la terapia cognitiva-conductual. Este enfoque promueve el desarrollo de nuevas habilidades de afrontamiento a los problemas a través de un aprendizaje activo. En este sentido, "las asignaciones para la casa representan una oportunidad para transferir las habilidades y las ideas del ámbito de la terapia a las situaciones cotidianas donde realmente ocurren sus problemas." (Manrique, 2014, p. 208). Se trabajaron los componentes del análisis funcional (Situación, pensamientos, emociones, conducta y consecuencias) y su relación. Luego, se presentaron y 
practicaron técnicas específicas para los componentes cognitivos, emocionales y conductuales (Ver Apéndice B).

\subsection{Intervenciones individuales}

Las intervenciones individuales se trabajaron siguiendo principalmente un enfoque cognitivo-conductual mediante sus diferentes tratamientos y técnicas. Sin embargo, como reconocen Ruiz, Díaz y Villalobos (2012) actualmente es aceptado la incorporación, en la práctica clínica y en los planes de intervención, de técnicas de diferentes orientaciones basadas en su valor pragmático.

Con la primera entrevista, se iniciaba el análisis del caso. Se anotaban los datos básicos del cliente y se discutía el motivo de consulta. De esta manera se comenzaba la evaluación del problema mediante la recogida de información relevante, teniendo en cuenta la historia del desarrollo del mismo, su expresión en diferentes contextos y sus componentes básicos. Las herramientas utilizadas para la evaluación de los problemas fueron las entrevistas semiestructuradas, el análisis funcional, autoregistros, autoreportes y fuentes secundarias (a disponibilidad) (Ver Apéndice C).

De acuerdo a Beck, Rush y Shaw (2010), bajo el enfoque de la terapia cognitivaconductual, desde la primera sesión se busca que el cliente comprenda el fundamento teórico de este tipo de intervenciones. Así, se explicaba en la primera consulta la relación entre pensamientos, sentimientos y conductas. Se dejaba para desarrollar en casa autoregistros específicos de la conducta problema y anotar los objetivos a alcanzar.

Después de la primera consulta y con los datos obtenidos se trabajaba una hipótesis del problema, la cual era utilizada como guía para las siguientes sesiones. En la 
segunda sesión, se continuaba con la recolección de datos, posibles comorbilidades, se revisaban los registros de las actividades asignadas, se discutían los problemas o dudas que podrían haber surgido y los objetivos a lograr. De esta forma, los aspectos que se trataban con los pacientes en las primeras sesiones eran: la base teórica de las intervenciones del enfoque cognitivo-conductual; la relación de los pensamientos, emociones y conductas y las metas y objetivos de la intervención.

Entre los casos que se presentaban, los que tenían trastornos depresivos y de ansiedad fueron los de mayor ocurrencia. De manera general, considerando las diferencias individuales y la conceptualización de cada caso con las personas con trastornos depresivos se trabajó: a) las dificultades conductuales; b) la motivación; c) los patrones y el contenido de pensamiento; c) las distorsiones cognitivas; y d) las actitudes y creencias. Utilizando las herramientas de programación de actividades, tareas de dominio-agrado, la identificación y desafío de pensamientos automáticos y las autoafirmaciones.

Con los problemas de ansiedad se trabajaron: a) el patrón de pensamiento; b) los pensamientos automáticos o distorsiones cognitivas; c) las estrategias de afrontamiento disfuncionales; d) las creencias de vulnerabilidad; e) los temores; y f) la tolerancia a la ansiedad. Utilizando la reestructuración cognitiva, técnicas de respiración y relajación, técnicas de exposición y técnicas de identificación y desafío de pensamientos automáticos.

También se atendieron problemas de dolor crónico, baja autoestima, habilidades sociales y relacionadas a la toma de decisiones.

Ante problemas de conducta en niños, se trabajaron con los padres de familia o cuidadores pautas de crianza y técnicas de modificación de conducta. 


\section{CAPÍTULO III: RESULTADOS DE LA INTERVENCIÓN}

El proyecto del Departamento de Psicología por sus objetivos y servicios ofrecidos puede ser considerado un programa de intervención clínico-comunitaria. El aporte de las actividades y tareas descritas anteriormente responden a lo esperado en este tipo de intervenciones.

Las intervenciones ejecutadas estuvieron sujetas a la evaluación y supervisión de los psicólogos profesionales encargados del Departamento de Psicología. En las sesiones de supervisión para las intervenciones individuales, se presentó y discutió a profundidad el proceso de recolección de datos, las herramientas de evaluación, el análisis funcional de cada caso y las técnicas trabajadas. Así, fueron aprobados y validados los planes de acción formulados. Como refiere Watkins (2018) conjuntamente con el desarrollo/mejora de las habilidades conceptuales/de tratamiento y de la identidad como psicoterapeuta, la supervisión también tiene una función de monitoreo y protección del bienestar de los clientes que reciben tratamiento por los supervisados (American Psychological Association, 2015; Beinart \& Clohessy, 2017; Falender \& Shafranske, 2017) (p.522). Se atendieron 57 casos de dos o más sesiones y las intervenciones grupales tuvieron un alcance de 60 personas. Se completaron seis programas individuales de consejería para padres centrados en las dificultades en la crianza y problemas de conducta de los hijos; cuatro intervenciones de consejería centrados en la resolución de problemas y cinco intervenciones psicológicas individuales. El 26.3\% de los casos atendidos llegaron a completar los programas de intervención psicológica individual. Los cinco clientes que completaron el proceso psicológico individual presentaron: a) trastorno de depresión con ansiedad por fibromialgia; b) agorafobia con ansiedad social; c) trastorno de adaptación con predominio de alteraciones de otras emociones (2); y d) trastorno de la personalidad dependiente. Cuatro de los cinco casos presentaron dificultades relacionados a la ansiedad o el estrés, lo cual refuerza la utilidad de la terapia cognitivo conductual en este tipo de afecciones. En estos casos, se utilizaron los registros semanales de 
las situaciones ansiógenas con autoevaluación de la intensidad de las respuestas emocionales. Asimismo, se trabajaron con todos técnicas de respiración y relajación, de reestructuración cognitiva e identificación de pensamientos distorsionados.

En repetidas ocasiones, los usuarios previamente atendidos pero que faltaron a las sesiones acordadas se presentaron en el consultorio para retomar las sesiones. En esos casos, de haber disponibilidad, fueron reprogramados y se hacía un pacto verbal de asistencia continua a las sesiones. Al explorarse los motivos de su inasistencia se encontró que a) habían tenido problemas de salud; b) tuvieron dificultades en su trabajo y/o estudios; y c) notaron una mejoría en cuanto a sus motivos de consulta después de algunas sesiones y antepusieron otras actividades/necesidades. El impacto de las intervenciones en los clientes fue medido de manera particular, utilizando los autoregistros, tareas y las autoevaluaciones que cada paciente reportó durante el periodo asistido. Las asignaciones para la casa facilitan la práctica regular de los nuevos comportamientos, lo cual fortalece la generalización de los mismos en su entorno diario.

Tanto en la sesión sobre las relaciones sexuales y las enfermedades de transmisión sexual como en el taller sobre drogas con los estudiantes adolescentes la asistencia fue total. Sin embargo, es posible que factores externos a la motivación propia de los adolescentes puedan haber influido en este aspecto dado que su asistencia fue requerida por la propia institución educativa. Asimismo, ambos fueron implementados de acuerdo a las necesidades encontradas en la población objetivo.

En cuanto a la implementación del taller de control de emociones, este respondió a la demanda del servicio de atención psicológica, los recursos disponibles y la orientación comunitaria del proyecto. A las personas que acudieron al Departamento de Psicología para recibir atención individual, que fueron entrevistadas y evidenciaron dificultades en el control de emociones, se les ofreció la posibilidad de participar del taller. El taller contó con 10 
inscritos inicialmente. En la segunda sesión se aceptaron dos participantes más, quienes fueron referidos por un participante del taller y un psicólogo del Departamento de Psicología. Sin embargo, cuatro de ellos tuvieron una asistencia irregular a las sesiones y uno se retiró del taller. Al evaluar los motivos de sus faltas, se encontró que habían tenido dificultades por el trabajo, los estudios y conflictos familiares. El $91.7 \%$ de los participantes terminaron el taller sin embargo, el $36.4 \%$ faltaron a una o más sesiones. 


\section{CONCLUSIONES}

- Las carencias que presentan los servicios de salud mental del país, la estigmatización de los problemas mentales y el desconocimiento de los mismos influyen significativamente en la búsqueda y recepción de asistencia en salud mental. Por lo cual, es de gran necesidad ampliar los conocimientos de la población mediante intervenciones de promoción y prevención en salud mental.

- El centro comunitario ofrece servicios educativos, médicos y sociales, los que son utilizados en su mayoría por vecinos del distrito en el que está ubicado. El cual presenta niveles importantes de pobreza.

- El trabajo realizado como voluntaria en el Departamento de Psicología consistió en brindar asistencia psicológica individual e intervenciones grupales de prevención y promoción de la salud mental.

- Durante el periodo laborado, el logro de objetivos y la adecuación de la metodología utilizada en las tareas incurridas se dio de manera cualitativa y personal. La constante supervisión por parte de un psicólogo profesional, los autoreportes de los pacientes y la observación de la conducta en sesión fueron las herramientas utilizadas para la valoración de la calidad de las intervenciones y su adecuación para los problemas presentados.

- La mayor dificultad encontrada fue la asistencia a los programas psicológicos individuales y al taller de mayor duración. Lo cual obstaculizó el seguimiento y medición del impacto de las intervenciones. Sin embargo, la Terapia Cognitiva Conductual permite, por medio de los autoregistros y tareas observar el avance en la adquisición de las nuevas conductas y sus consecuencias.

- Existe poca información e investigación sobre la práctica de la psicología clínica en el Perú. Lo cual dificulta la medición y contrastación de las intervenciones en salud mental ajustadas a la realidad nacional. 


\section{RECOMENDACIONES}

A continuación se detallaran las recomendaciones:

- Es usual que los usuarios de los servicios del Departamento de Psicología presenten dificultades para mantener una asistencia regular a las sesiones y culminar con los programas de intervención terapéutica propuestos. Al evaluar los motivos de esta irregularidad, se encuentra que son situaciones externas a la intervención lo que los lleva a desistir. La inclusión de un cuestionario para la medición de la satisfacción con los servicios, podría brindar indicadores sobre el impacto de las intervenciones, a pesar de la variabilidad en la asistencia de los usuarios.

- Mantener una evaluación constante de los voluntarios del proyecto social que les permita notar los aprendizajes adquiridos durante su participación. De acuerdo a Hombrados (2013) es posible que se fomente el apoyo y permanencia de los voluntarios si perciben que sus tareas están bien definidas, se les brinda orientación y supervisión constante y que están recibiendo formación. Dado que el proyecto funciona en base a voluntarios, tener una guía sobre su desempeño y recibir sesiones de supervisión podría generar mayor motivación para mantener su labor en el proyecto.

- Crear una base de datos con la información de los casos atendidos para aumentar el conocimiento acerca de la población usuaria del Departamento de Psicología. Asimismo, se podrían generar guías basadas en evidencia sobre los problemas de mayor prevalencia que podrían ser de utilidad para la capacitación de los voluntarios ingresantes y demás colaboradores. 


\section{REFERENCIAS}

Ardila Serrano, L. M. (2007). Adolescencia, desarrollo emocional: Guía y talleres para padres y docentes. Bogotá, D.C: Ecoe.

Barrio Gándara, V. d. (2016). Evaluación Psicológica. (Segunda. ed.). Madrid: Centro de Estudios Financieros.

Beck, A. T., Rush, A. J. y Shaw, B. F. (2010). Terapia cognitiva de la depresión (19a. ed.).

Calero, A., Schmidt, V., y Bugallo, L. (2016). Consumo de alcohol y su relación con la autopercepción adolescente. Salud y drogas, 16(1), 49-58. Recuperado de: https://www.redalyc.org/articulo.oa?id=83943611005

Giraldo Osorio, A., Toro Rosero, M., Macías Ladino, A., Valencia Garcés, C., \& Palacio Rodríguez, S. (2010). La promoción de la salud como estrategia para el fomento de estilos de vida saludables. Revista Hacia la Promoción de la Salud, 15(1), 128-143. Recuperado de: https://www.redalyc.org/articulo.oa?id=309126693010

Hombrados, M. M. I. (2013). Manual de psicología comunitaria. Madrid: Síntesis.

Instituto Integración de análisis y comunicación. (2016). Artículo: 80\% de peruanos con problemas de salud mental no son atendidos. Recuperado de: http://www.integracion.pe/80-peruanos-problemas-salud-mental-no-atendidos/

Instituto Nacional de Estadística e Informática (INEI), 2017. Censos Nacionales 2017: XII de Población y VII de Vivienda. Recuperado de https://www.inei.gob.pe/

Instituto Nacional de Estadística e Informática (INEI). 2018. Censos Nacionales 2017: XII de Población y VII de Vivienda. Perú: Crecimiento y distribución de la población, 2017. Primeros Resultados. Recuperado de: https://www.inei.gob.pe/media/MenuRecursivo/publicaciones_digitales/Est/Lib1530 /libro.pdf 
Instituto Nacional de Estadística e Informática (INEI). (2015). Mapa de Pobreza Provincial y Distrital 2013. Recuperado de: https://www.inei.gob.pe/media/MenuRecursivo/publicaciones_digitales/Est/Lib1261 /Libro.pdf

Lavado, P., Aragón, C., y Gonzales, M. (2015). ¿Cuál es la relación entre las habilidades cognitivas y no cognitivas y la adopción de comportamientos de riesgo? un estudio para el Perú. Apuntes, 42(76), 59-93. Recuperado de: http://www.proquest.com

Manrique, G. E. (2014). Cultura, Cognición Social y Terapia Cognitivo Conductual.Lima: Taller Gráfico Guilse.

Ministerio de Salud (MINSA). (Junio, 2018). Plan Nacional de Fortalecimiento de Servicios de Salud Mental Comunitaria 2017 - 2021. Recuperado de: http://bvs.minsa.gob.pe/local/MINSA/4422.pdf

Organización Mundial de la Salud (OMS). (Diciembre, 2013). OMS | Salud mental: un estado de bienestar. Recuperado de: http://www.who.int/features/factfiles/mental_health/es/

Organización Panamericana de la Salud (2009). Estrategia y plan de acción sobre salud mental. Recuperado de: http://www1.paho.org/hq/dmdocuments/2009/SALUD_MENTAL_final_web.pdf.

Ruiz, F. M. A., Díaz, G. M. I., \& Villalobos, C. A. (2013). Manual de técnicas de intervención cognitivo conductuales. Bilbao: Desclée De Brouwer.

Sánchez, V. A. (2007). Manual de psicología comunitaria: Un enfoque integrado. Madrid: Pirámide.

Watkins, C. E., Jr. (2018). The Generic Model of Psychotherapy Supervision: An AnalogizedResearch-Informing Meta-Theory. Journal of Psychotherapy Integration, 28(4), 521-536. doi: 10.1037/int0000114 


\section{APÉNDICES}


APÉNDICE A: Esquema de la sesión con adolescentes sobre las relaciones sexuales y enfermedades de transmisión sexual 


\section{Tabla A.}

Esquema de la sesión con adolescentes sobre las relaciones sexuales y enfermedades de transmisión sexual

\begin{tabular}{|c|c|c|c|c|}
\hline Duración & Actividad & Objetivo & $\begin{array}{l}\text { Descripción de actividades y desarrollo de } \\
\text { ideas }\end{array}$ & Medios y materiales \\
\hline 5 & Presentación & $\begin{array}{l}\text { - Conocer los nombres } \\
\text { de los participantes y } \\
\text { del facilitador. } \\
\text { - Presentar el tema a } \\
\text { abordar }\end{array}$ & $\begin{array}{l}\text { Se entrega una etiqueta en la cual los } \\
\text { adolescentes escriben su nombre } \\
\text { Se brinda una presentación general del } \\
\text { facilitador y del tema a sobre el cual se } \\
\text { trabajará. }\end{array}$ & - Etiquetas \\
\hline $25^{\prime}$ & $\begin{array}{c}\text { Técnica } \\
\text { "Emparejando" }\end{array}$ & $\begin{array}{l}\text { Reconocer las } \\
\text { principales ETS y sus } \\
\text { vías de transmisión }\end{array}$ & $\begin{array}{l}\text { Se dividen a los adolescentes en } 2 \text { grupos de } \\
\text { trabajo. Se les reparten las etiquetas. } \\
\text { La actividad consiste en emparejar el nombre } \\
\text { de la ETS con sus síntomas y vías de } \\
\text { transmisión. } \\
\text { Se contrastan y discuten las respuestas entre } \\
\text { ambos grupos. } \\
\text { Se brinda información complementaria y se } \\
\text { resuelven dudas }\end{array}$ & $\begin{array}{l}\text { - Etiquetas con los nombres de las } \\
\text { ETS (Clamidia, VIH/HIV, Herpes, } \\
\text { Gonorrea, VPH, Sífilis) y otras con } \\
\text { sus vías de transmisión y síntomas. } \\
\text { - Pizarra, limpiatipos }\end{array}$ \\
\hline $10^{\prime}$ & $\begin{array}{c}\text { Técnica "lluvia } \\
\text { de ideas" }\end{array}$ & $\begin{array}{l}\text { Identificar los riesgos } \\
\text { en las relaciones } \\
\text { sexuales }\end{array}$ & $\begin{array}{l}\text { Se hace la pregunta: ¿Cuáles son los posibles } \\
\text { riesgos que uno puede encontrar al mantener } \\
\text { relaciones sexuales? } \\
\text { Un adolescente voluntario apunta en la }\end{array}$ & $\begin{array}{l}\text { - Cartulinas } \\
\text { - Plumón }\end{array}$ \\
\hline
\end{tabular}


cartulina los riesgos que junto a sus

compañeros identifican.

- Educar en métodos A partir de los riesgos encontrados se expone 
APÉNDICE B: Esquema del taller manejo de emociones 


\section{TALLER MANEJO DE EMOCIONES}

\section{Sesión 1:}

- Descripción de las emociones, clasificación de ellas (agradables (alegría, sorpresa, felicidad, amor, amistad), desagradables (tristeza, cólera, miedo))

- Explicación de relación entre situación y emoción. Se brindan ejemplos y cada uno va proponiendo algunos de ellos (p.ej. me peleo con mi pareja - me da tristeza)

\section{Sesión 2:}

- Repaso de lo descrito la sesión anterior (situación y emoción)

- Se describe la importancia del pensamiento entre la situación y la emoción

- Se conversa que los pensamientos aparecen y generan que también se sientan las emociones de diferentes maneras

- Se divide el salón en grupos para plantear ejemplos, y propongan ejercicios

\section{Sesión 3:}

- Repaso de lo descrito la sesión anterior (situación-pensamiento-emoción)

- Se piden que den ejemplos sobre estos 3 elementos.

- Se agrega las conductas y las consecuencias

- Brindar un ejemplo por equipo sobre los 5 elementos en acción

- Se deja como tarea completar una hoja de autoobservación

\section{Sesión 4}

- Repaso de todos los elementos del análisis funcional

- Se explica el trabajo en cada uno de los 3 elementos centrales (pensamiento-emoción y conducta). Se inicia el trabajo con la parte emocional a través de una técnica de respiración y relajación

- Se enseña la respiración diafragmática (llevar el aire al estómago)

- Se desarrolla una sesión breve de relajación imaginal

- Se deja como tarea practicar tanto la respiración como la relajación en casa

\section{Sesión 5}

- Repaso de la respiración y relajación (se hace la práctica nuevamente, además de proponer esta relajación prestando atención a partes de nuestro cuerpo)

- Se explica la importancia del trabajar los pensamientos

- Descripción a través de ejemplos lo que son pensamientos racionales vs. irracionales

- Tanto en equipos como de manera individual se proponen pensamientos racionales e irracionales de acuerdo a sus experiencias y a ejemplos propuestos por los facilitadores

\section{Sesión 6}

- Repaso de la respiración y relajación (practica a través de imaginación y prestar atención a estímulos externos) 
- En grupos, comparten los pensamientos racionales e irracionales que proponen en la tarea. Se repasa lo conversado la sesión anterior

- Se les explica 5 ejemplos de pensamientos irracionales: filtraje, pensamiento polarizado, visión catastrófica, deberías, sobregeneralización.

- A través de hojas de trabajo, identifican sus pensamientos asociados a situaciones.

- Se deja como tarea que describan esos pensamientos con las situaciones asociadas.

\section{Sesión 7}

- Repaso de los pensamientos distorsionados vistos en sesión anterior.

- Se explican otros pensamientos irracionales que pueden existir: ¿y si?, deberías, razonamiento emocional, comparación a lo negativo. Se piden que brinden ejemplos personales, para realizar una discusión. Se hablan de las consecuencias negativas que tienen esos pensamientos en sus vidas.

- Se hace actividad del espejo: en la parte externa redactar todos los pensamientos irracionales y negativos que tienen, y dentro del espejo todos los pensamientos positivos que se podrían decir sobre sí mismos.

- Se les deja como tarea cortarlo, y quedarse con el espejo con las cosas positivas, y que lo guarden y lo peguen en un lugar visible.

\section{Sesión 8}

- Revisión de los pensamientos distorsionados vistos en la sesión anterior

- A través de preguntas de utilidad, ventajas vs. desventajas, pros y contras, se inicia con la reestructuración.

\section{Sesión 9}

- Revisión de la reestructuración propuesta

- Planificación de actividades que sean placenteras y de agrado, en función a las necesidades de cada paciente

\section{Sesión 10}

- Revisión de actividades.

- Repaso general de lo visto durante todas las sesiones

- Cierre del taller

Nota: Este esquema fue utilizado para desarrollar el taller de control de emociones en el proyecto social bajo la supervisión del autor del mismo. 
APÉNDICE C: EJEMPLO DE ANÁLISIS FUNCIONAL DE UN CASO 
Tabla C.1.

Ejemplo de Análisis Funcional de un caso

Ed

O-R

Er

\section{Externos}

- Al mirar videos o escuchar música que hablan sobre tiempos pasados felices y de soledad.

- Al enfrentar una situación social ansiógena.

- Al ver familias riendo y pasando tiempo juntos.

- Al interactuar con su padre.

- Al intervenir en la relación de sus padres.

\section{$\underline{\text { Internos }}$}

- Al recordar situaciones en las que considera haber fallado (su vida familiar, educativa, social)

- Al pensar en situaciones en las que no fue capaz de dar su opinión con certeza o
Motor - conductual

- Evitación de situaciones sociales

- Llanto frecuente

\section{$\underline{\text { Cognitivo }}$}

Sobre sí misma: "Soy una inútil, no puedo hacer nada", "Soy una estúpida", "No tengo habilidades para nada y dependo de otros para todo", "Toda mi vida me he equivocado.", "Soy muy rara"

Sobre el futuro: "Nada va a cambiar, tengo mucho miedo a fracasar", "Necesito saber cómo va a salir todo para recién empezar a pensar en qué hacer", "Nunca vas a mejorar. Van a pasar siglos y vas a seguir haciéndolo mal", "Preferiría morir antes de seguir así".

Sobre los otros: "Soy un fracaso, me van a

\section{Externos}

- Pocas obligaciones en casa.

- Pocos pasatiempos/actividades de interés y bajo interés en realizarlas

- Madre la acompaña a dónde desee ir y al mismo tiempo la regaña por no poder hacer las cosas sola.

- Reprensión constante del padre por haber salido de la universidad y comentarios desmotivadores y predictores de fracaso.

$\underline{\text { Internos }}$

- Rumiación sobre sus intervenciones en situaciones sociales que considera fallidas y de decisiones pasadas. Pensamientos de desesperanza, comparación con otras 
decidir por sí misma.

- Al recordar las palabras de su madre "La única razón por la que has terminado el colegio en un buen puesto ha sido por mí. Si no, no lo podrías haber hecho"

- Al pensar en enfrentar una situación ansiosa.

- Recordar su actuación en una situación social ansiógena. juzgar, mejor me quedo callada." "Si puedo personas de su edad,

evitar hablar con alguien, mejor. No quiero - Bajo autoconcepto, baja autoeficacia.

molestar y hacer una pregunta estúpida. De - Evitación de situaciones y de amistades.

ahí se dan cuenta que soy una idiota", "Es

mucho esfuerzo estar con otros, socializar.

Prefiero estar en mi cuarto con mi gato".

Fisiológico - emocional

- Dolores de cabeza

- Fatiga - Culpa

- Insomnio

- Tristeza/Estado de ánimo depresivo

- Problemas de concentración

- Irritabilidad

- Indecisión

Dimensiones de la conducta

- Frecuencia: Diaria

- Duración: Variable

- Intensidad: Alta 